\title{
Initial jitter analysis of Lynx, a proposed future large Astrophysics Facility
}

\author{
J. Brent Knight*, Jessica A. Gaskin*, Alex Dominguez*, Jay Garcia* \\ *NASA Marshall Space Flight Center, AL, USA 35812
}

\begin{abstract}
Lynx, formerly known as the X-Ray surveyor, is one of the large strategic mission concepts being studied for input into the 2020 Astrophysics Decadal Survey. Lynx is the first future X-ray mission concept planning to match Chandra's angularresolution and will combine this with very high throughput, large field of view, and high-resolution spectroscopy for pointlike and extended sources. These ambitious performance requirements clearly merit early detailed engineering to demonstrate feasibility. An on-going structural dynamic analysis is being performed on the Lynx structural design to predict dynamic responses, jitter, to expected on-board vibrational disturbances. Applicable disturbance sources include a cryogenic pump, and six reaction wheels. The structural design, disturbances, analysis, and results are presented. Ultimately, responses will be compared to Lynx performance requirements as they relate to a system error budget.
\end{abstract}

Keywords: X-Ray Telescope, Jitter, Stability, Line of Sight

\section{INTRODUCTION}

All space telescopes are confronted with notable engineering challenges. This is perhaps most true for those telescopes that are planned for a future launch, as concepts are developed to meet ever more ambitious science goals. Nearly "perfect" in-service alignment and stability are paramount for performance of space optics systems. One potential impact to performance is jitter, which, in this paper, are the dynamic responses/motion due to on-board vibratory sources (reaction wheels, cryocooler, etc.). Figure 1 presents the current conceptual design. The global structural design uses M46J composite material, but M55J is also being investigated.
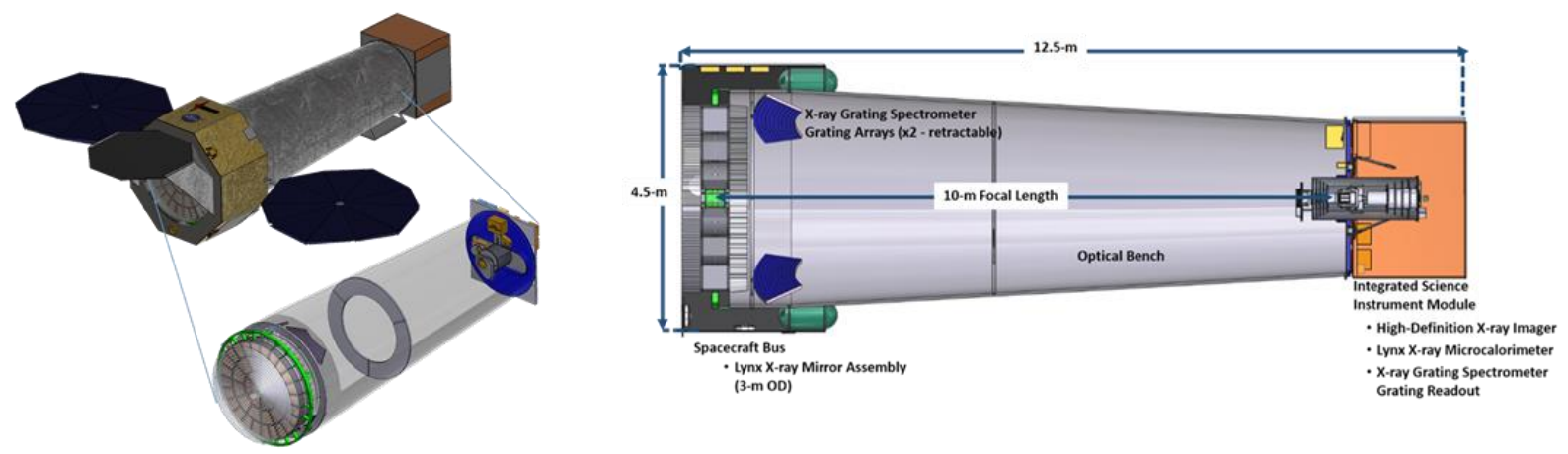

Figure 1. Current Lynx Conceptual Design 
A structural dynamic Finite Element Analysis (FEA) was performed on the current Lynx design to estimate the order of magnitude of expected jitter during normal mode on-orbit operation. Lynx is expected to have two vibration excitation sources during this operational mode, rotating imbalance of the six Reaction Wheel Assemblies (RWA) and a cryocooler. Only the non-isolated RWAs were considered in this analysis. Chandra specified maximum allowable RWA vibration levels were used as the analytical forcing functions (dynamic loads). Future analyses are planned to assess the effect of the cryocooler once more detail is defined for pertinent interfaces. The combination of all disturbances on the telescope on-orbit performance will then be predicted as well.

The objective of the efforts documented here is to identify global design parameters that can influence the order of magnitude of expected jitter. Concept design parameters that could be influenced by results of this type include:

\author{
Material type/properties \\ Load paths \\ Disturbance sources \\ Locations \\ Types \\ Control systems \\ Potential need for isolation \\ Design personnel allocate volume for isolation systems
}

Invariably, space optical systems pose performance requirements that are engineering challenges. Jitter is known to be an impact to system performance. Therefore, even at this very early stage, it is prudent to address pertinent engineering to the degree meaningful.

\title{
2. FINITE ELEMENT MODEL AND FORCING FUNCTIONS
}

The current Lynx Finite Element Model (FEM) consists of 80,351 elements, 85,733 nodes, and 419 multi-point constraints (MPC). The elements are vastly shell elements. Table 1 presents the NASTRAN standard FEM summary that is provided in analysis output. The "ENTRY NAME" items in the table are the various types and quantities of elements, properties, materials and grids.

Table 1. NASTRAN Summary of the Lynx FEM

$\begin{array}{cr}\text { M O D E L S U M M A R Y } \\ \text { ENTRY NAME } \\ \text { NUMBER OF ENTRIES } \\ \text { CBAR } & 2286 \\ \text { CHEXA } & 10 \\ \text { CONM2 } & 9 \\ \text { CQUAD4 } & 77846 \\ \text { CTRIA3 } & 200 \\ \text { EIGRL } & 1 \\ \text { GRID } & 85733 \\ \text { MAT1 } & 9 \\ \text { MAT2 } & 24 \\ \text { MAT8 } & 3 \\ \text { PARAM } & 4 \\ \text { PBARL } & 7 \\ \text { PCOMP } & 67 \\ \text { PSHELL } & 19 \\ \text { PSOLID } & 1 \\ \text { RBE2 } & 23 \\ \text { RBE3 } & 396\end{array}$


The FEM utilized was developed by NASA MSFC's Advanced Concepts Office (ACO) for initial frequency predictions and stress analyses. It includes representation of a Space Craft (SC), a conceptual design of the optical bench, and a concentrated mass to represent the mirror (the mass of the mirror is mathematically applied at a single point and it's connected to supporting structure). Non-structural mass was incorporated to represent spacecraft elements such as avionics boxes, batteries, fuel, etc. The FEM, at this point in time, does not include representation of the solar panels which in service could pose dynamics engineering challenges. The next FEM iteration is expected to include a first cut representation of the solar panels.

Mathematical representations of external loads and/or environments have to be applied in any structural analysis. In dynamic structural analyses, those loads are often referred to as "Dynamic Forcing Functions" (DFF). Ultimately, these loads or DFF's are numerical representations of the condition that the structure is assessed to. In this analysis, to predict jitter, that external load is the vibration created by a RWA applied at six locations. The DFF utilized in this analysis is the Chandra RWA vibration specification data. That specification required that the vibrations due to the RWA rotating imbalance were below a required limit (Figure 2). Since Chandra satisfied that requirement this is a conservative assumption. With respect to Guidance Navigation and Control (GNC), once the observatory is pointed at its target and transients due to re-positioning are diminished, only RWAs and a cryocooler (the latter is not included in this analysis) are expected to be sources of vibration. Therefore, all excitations due to other GNC components are ground ruled out during normal-mode operation.

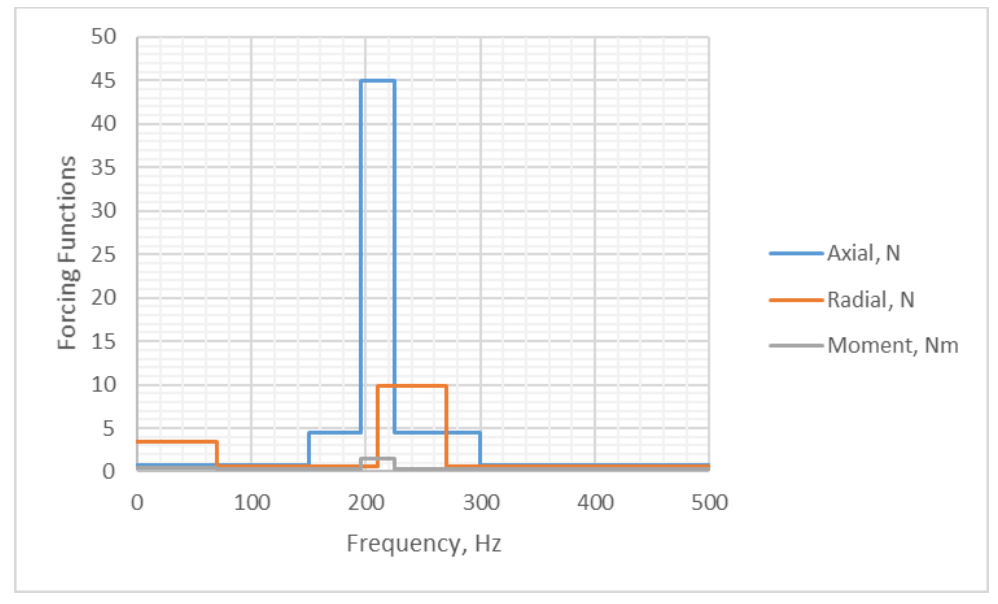

Figure 2. Forcing Functions

Figure 3 presents the integrated Lynx optical bench and spacecraft FEM as well as the analysis coordinate system. The Integrated Science Instrument Module (ISIM) houses the science instrument suite, while the Lynx X-ray Mirror Assembly (LMA) is housed inside the periphery of the spacecraft bus.

\section{ANALYSIS}

A structural dynamic analysis using MSC NASTRAN was performed using the described FEM and DFFs. Damping was assumed to be $1.0 \%$ and analyses were performed with the model in the free-free configuration (the FEM, mathematically, is not connected to anything as Lynx will be in-service). An overall Uncertainty Factor (UF) of 2.0 was applied to the results. It is possible that certain analysis assumptions can increase the effective UF but are not easily quantifiable. One example is the assumption that all RWA spin axes are aligned with the FEM's axial direction, when in reality they likely will not be. Another is the assumption that all six RWAs rotating eccentric mass are in-synch. The likelihood of six rotating mass having the orientation of their eccentric CGs lined up with one another is extremely remote. Use of an existing RWA specification as 
input is conservative since there are bands across the spectrum at which RWA performance is well below the requirement. With the UF of 2.0 combined with described inherent UFs, predictions are expected to be conservative. That is, higher than what Lynx would experience if this was the final design and Lynx specific DFFs. The current plan, with respect to RWA position and orientation, is to assume Chandra's configuration. Analytically, the DFFs input locations are shown in Figure 4.

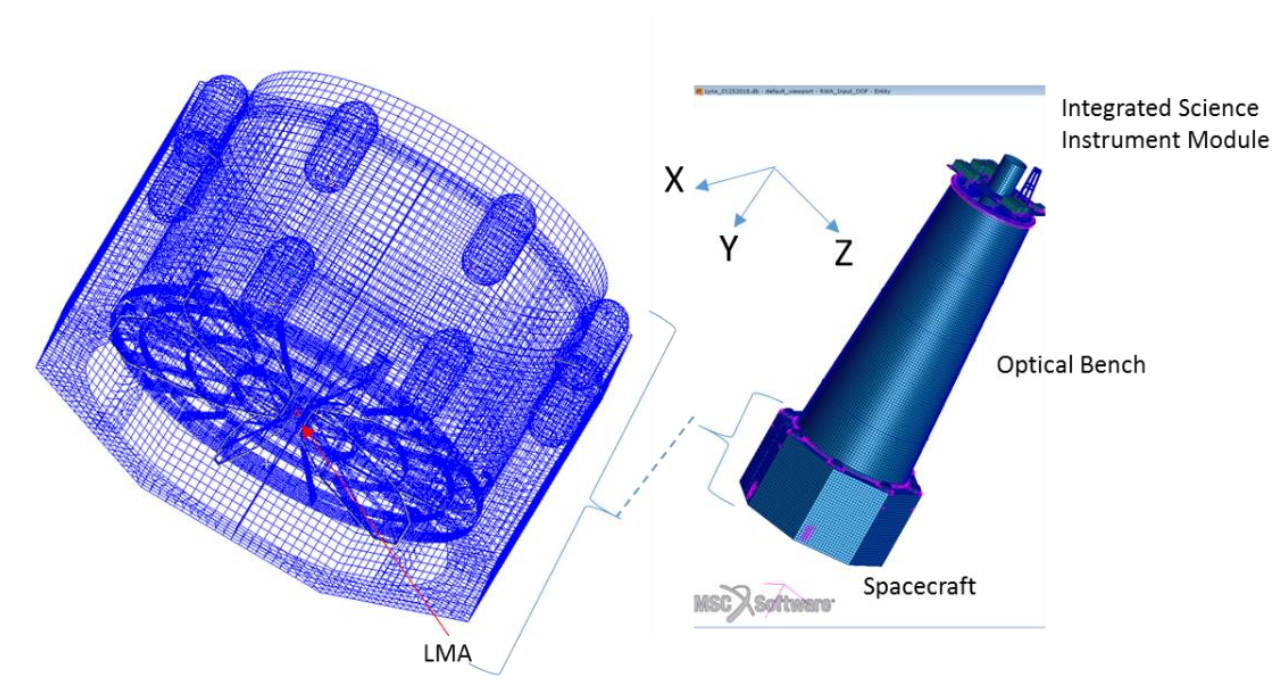

Figure 3. Current Lynx FEM

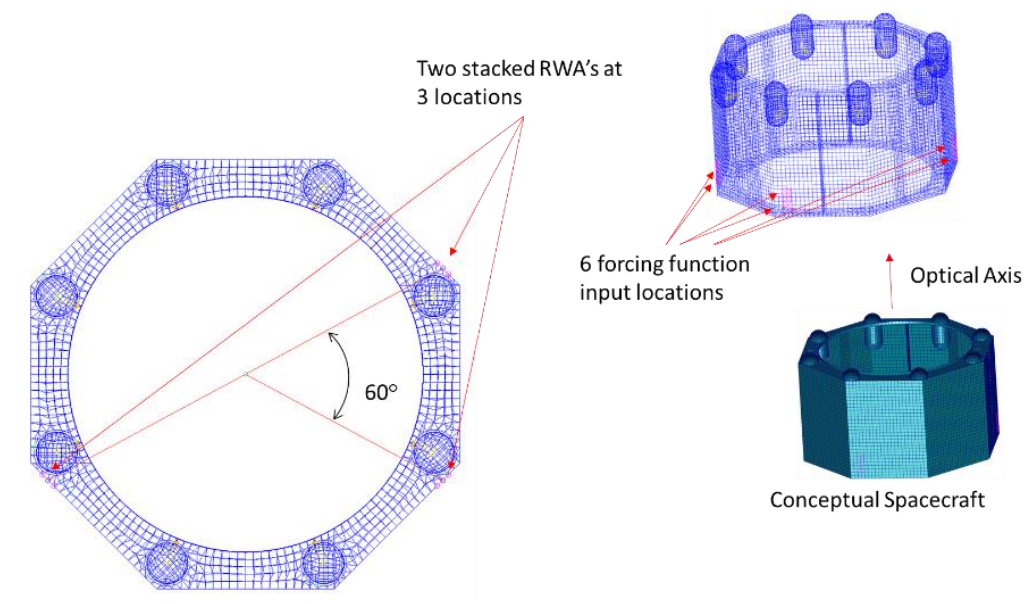

Figure 4. DFF Input Locations 
The inputs are in the form of frequency dependent forces and moments. Included are oscillating forces parallel to the wheel's shaft (axial) and radial (XZ-plane) as well as moments (M). The radial load is considered at two positions of its rotating cycle. The first position is when the force vector is parallel to the global $\mathrm{X}$ direction and the other parallel to the $\mathrm{Y}$ direction. Four cases of combined loads are assessed:

Radial $\mathrm{X}$ in global coordinates $+\mathrm{Mx}+$ Axial
Radial $\mathrm{X}$ in global coordinates $+\mathrm{Mz}+$ Axial
Radial $\mathrm{Z}$ in global coordinates $+\mathrm{Mz}+$ Axial
Radial $\mathrm{Z}$ in global coordinates $+\mathrm{Mx}+$ Axial

Load vector orientation is depicted in Figure 5. In each case, the three loads, at each of the six RWAs, are acting in phase, which is not realistic but serves as a first cut look at their non-isolated effect. The load vectors are incorporated as if the axis of each wheel is parallel to the optical axis, but in reality the RWAs will likely be canted similarly to Chandra's.

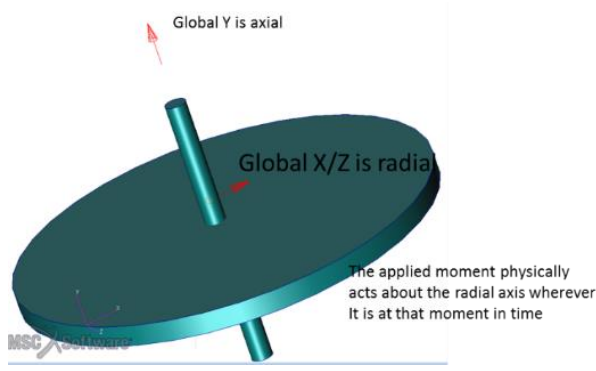

Figure 5. Applied Load Coordinates

A final point to make regarding differences between the physics of RWA vibratory loads, categorically rotating imbalances, and how they are represented mathematically is that inherent to a frequency response analysis the in-plane components of the radial load are not completely reflective of the phenomena being modeled. When, for example, the radial load vector is parallel with the $\mathrm{X}$-axis the Z-load is zero physically. However, within the identified bounds of performing a frequency response analysis utilizing FEA, no means to phase those has been identified. By including the loads in both $\mathrm{X}$ and $\mathrm{Z}$ mathematically, they would be in-sync while physically they are the opposite. When the X-component is at its maximum, the Z-component is zero. In a sense, to have the two components maxima in-sync would be perceived as conservative but if there are modes in a structure that are more sensitive to the radial load somewhere between the $\mathrm{X}$ and $\mathrm{Z}$ axes that may be missed. However, for this first assessment of Lynx with the design and FEM being very symmetric, doing so is considered an acceptable estimate. Future analyses will be performed via transient analyses to circumvent this effect.

A point at the currently anticipated center of the LMA (located inside the spacecraft bus periphery) as well as one near the center of the focal plane (ISIM) were selected as output locations to predict the relative motion between the two. The acceleration of the ISIM and LMA points were also predicted.

Displacements and accelerations at the LMA and ISIM Degrees Of Freedom (DOF) were output at $10.0 \mathrm{~Hz}$ intervals from 0 to $500 \mathrm{~Hz}$ and three additional frequencies associated with each modal frequency. Results for a total of 7,316 frequencies were output. The difference in displacement, or relative motion, between the corresponding DOF of those 2 points is considered an estimate of the line-of-site error due to RWA vibrations. Additionally, acceleration of the LMA and ISIM FEM points are 
considered an estimate of the vibration/acceleration input to those structures due to RWA vibrations. However, it should be noted that it does not represent the potential for local or individual mirror motion due to that disturbance. A future analysis will incorporate a detailed FEM of the LMA that will provide insight into the magnitude of the RWA vibration effect on the mirrors.

\section{RESULTS}

Envelopes of results from the four load cases analyzed are presented in Figures 6 through 8. Figure 6 presents the predicted relative motion between the LMA and the ISIM. Figures 7 and 8 present predicted acceleration at the LMA and the ISIM respectively. Those data are to be utilized as input in future analyses.

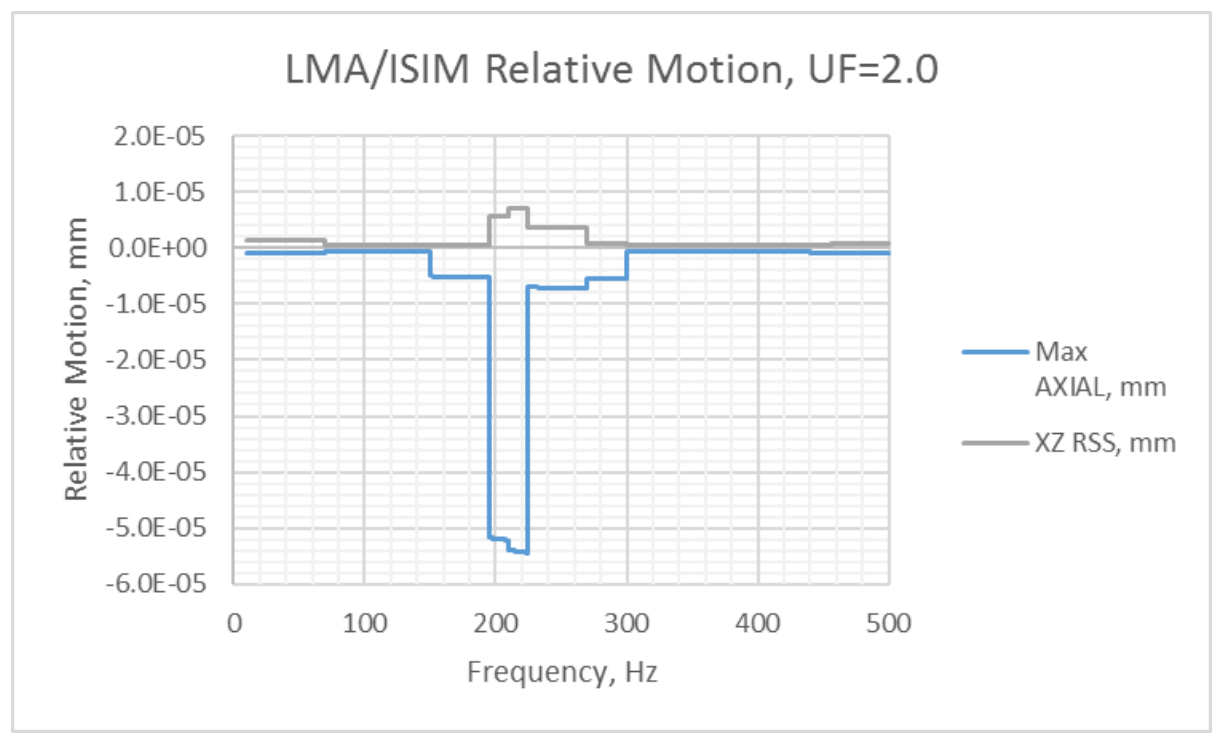

Figure 6. Relative Motion between the Focal Plane and the LMA 


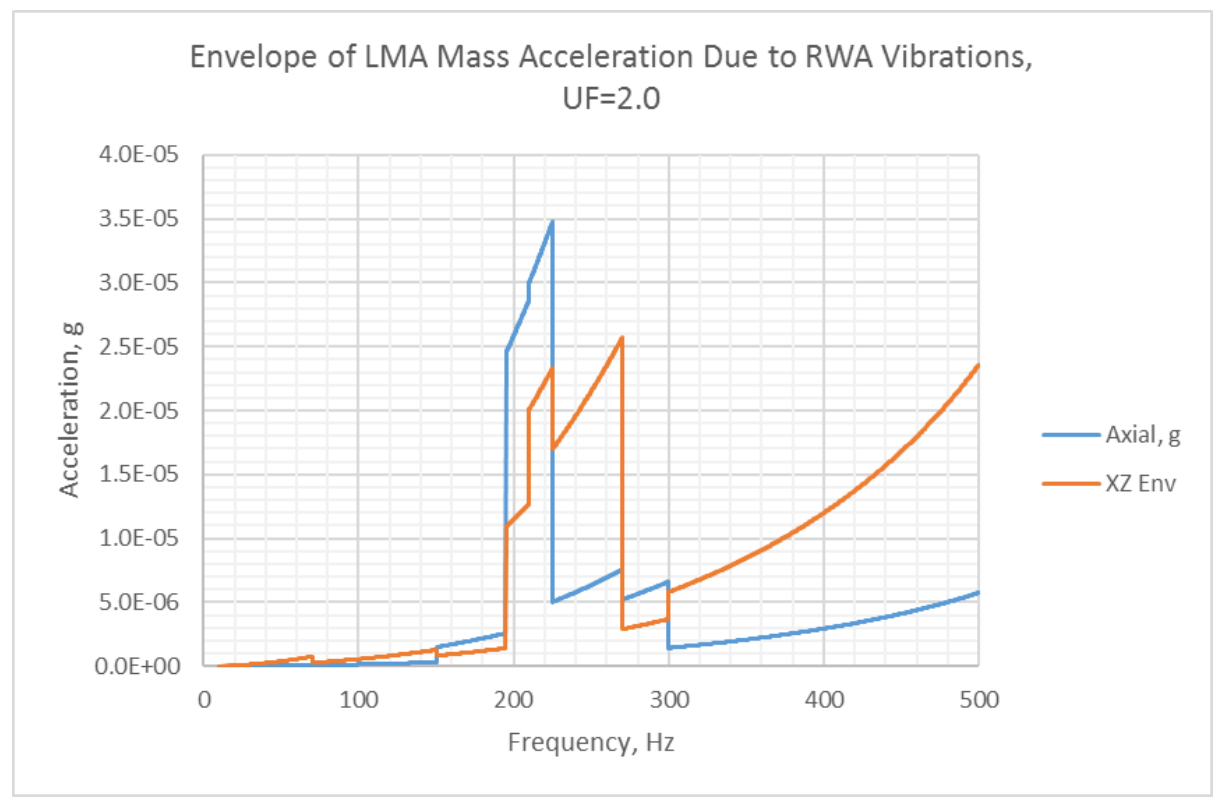

Figure 7. LMA Acceleration

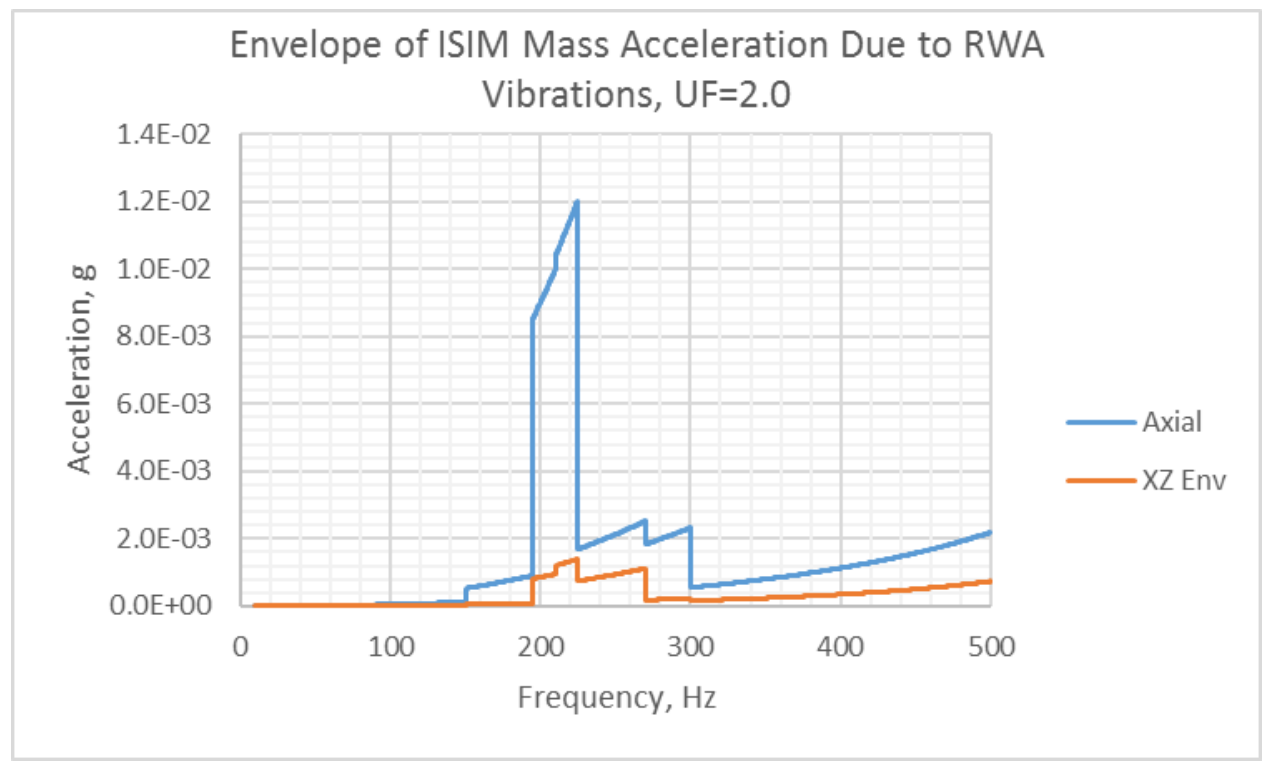

Figure 8. ISIM Acceleration

\section{DISCUSSION}

Mechanical misalignment of crucial telescope elements and all motion in service (thermal deformations and dynamic responses) will, to some degree, impact the system performance. This impact will be quantified through an integrated error budget analysis that will factor in these results, along with thermo-elastic analyses. In the event that the error budget for dynamic motion is less than what is predicted, vibration isolation or other design solutions will be pursued. 
Analyses documented in this paper are based on an early conceptual design and will continue to be refined as the concept fidelity increases. The current predicted dynamic motion of the points of interest are estimates, and represent a first best guess of the displacements Lynx will see on-orbit due to the RWAs. These analyses were performed, as will be near term future analyses, in support of feasibility and proposal type activities. The Lynx team is leveraging, to the greatest extent possible, Chandra's success. Results from these analyses will be utilized to demonstrate that dynamics challenges that Chandra incurred will be mitigated to the greatest extent possible.

The max current predicted relative motion between LMA and the ISIM is of the order of -5.5E-5 mm, occurring at $\sim 225 \mathrm{~Hz}$. Therefore, when the RWAs pass thru $\sim 225 \mathrm{~Hz}$, the largest jitter amplitude is expected. The duration of that max condition depends on the GNC control algorithm and the space environment. The negative sign on the displacement indicates that the LMA and ISIM points in the FEM are moving away from one another at that frequency and during that peak oscillating motion.

The acceleration results from this analysis currently offers no direct performance insight. Those data are intermediate results to be used in future analyses.

\section{CURRENT AND FUTURE WORK}

The following items are planned for follow-on near-term Lynx structural analyses associated with on-orbit performance:

1. Once the first cut error budget is in hand, results will be compared to that and the need or not for isolation will be addressed.

2. Analyses will be re-run using a different composite material, MJ55.

3. An LMA trade study to assess the Lynx Design Reference Mission mirror technology is currently underway. Once completed, a detailed design of the LMA using that technology will be incorporated into the Lynx observatory model. A FEM of the selected mirror will be made and incorporated into the integrated Lynx FEM. Detailed local LMA predictions will then be made, providing early order of magnitude estimates of individual mirror shell vibrations.

4. Analyses to determine the duration for motion to dampen out after the system is moved and re-pointed at a new target will be performed. This may result in designing in more damping.

5. Estimated vibration environments at optical component interfaces due to the cryocooler will be predicted and combined with the estimated vibrations from the RWAs to identify subsystem elements that may need isolation.

6. Thermo-elastic analyses will be performed utilizing predicted thermal gradients and models to estimate the amount of motion that is expected at the optical points of interest due to the space thermal environment. 